\section{COMPUTER MICROVISION FOR MEMS}

Dennis M. Freeman, Massachusetts Institute of Technology freeman@mit.edu

Computer Microvision is a microscopy technique originally developed to study sound-induced motions of sensory cells in the inner ear - motions that are on the order of picometers-i.e., smaller than the radius of a hydrogen atom. Three-dimensional micromovies of inner ear cells moving in response to sound were analyzed to quantify motions with nanometer precision. Recently we have adapted the technique for in situ measurement of motions of micro-electron mechanical (MEMS) devices.

In Computer Microvision systems, images of MEMS are magnified with an optical microscope and projected onto a CCD camera. Stroboscopic illumination is used to obtain stopaction images of the moving structures. Stop-action images from multiple focal planes provide information about 3D structure and 3D motion. Image analysis algorithms determine motions of all visible structures with nanometer accuracy. The system can measure motions at frequencies as high as $5 \mathrm{MHz}$ with nanometer resolution, i.e., well below the wavelength of light.

\section{Background}

Powerful tools have been developed to test electrical behaviors of microfabricated systems. By contrast, relatively few tools are available to measure micromechanical behaviors. This paucity of tools for measuring micromechanical behaviors has represented a critical obstacle to the widespread use of MEMS, because designers could not SEE the motions of the structures they build. As a result, mechanical problems may not be discovered until after many costly prototypes have been built. Also manufacturers are handicapped because mechanical testing may not be practical until late in the manufacturing process - perhaps not even until after packaging. To resolve such problems, we developed the Computer Microvision system so that a variety of surface and bulk micromachined struc-

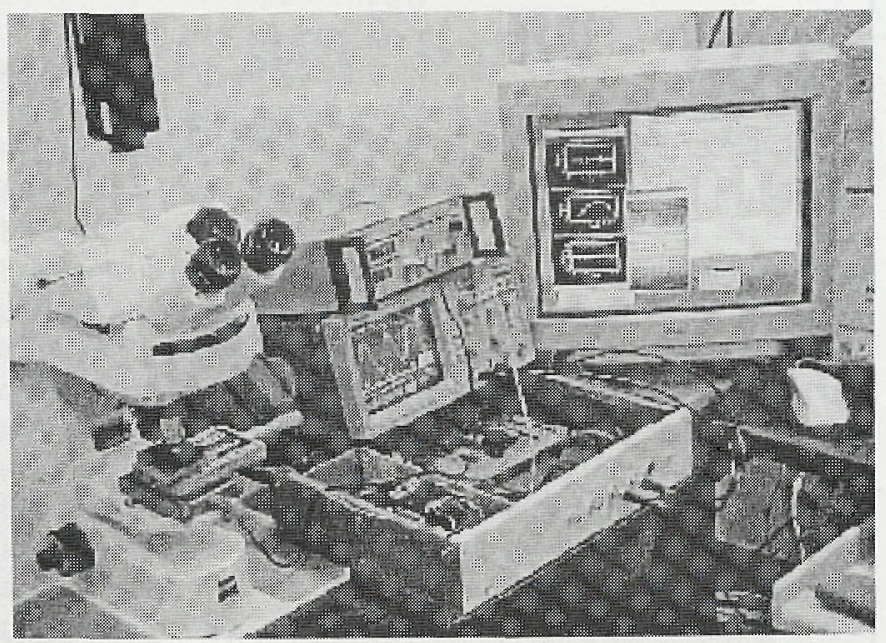

Figure 1: Computer Microvision measurement system. MEMS are positioned on the stage of a microscope (left) that has been modified for 3D stroboscopic imaging by (1) replacing the halogen lamp with an LED, (2) mounting the microscope objective on a piezoelectric focusing unit (controller shown in top center), and (3) attaching a CCD camera (top left). A computer controls a modular stimulus generator (bottom center) which provides two synchronized waveforms: one to drive motions of the MEMS and one to pulse the LED for stroboscopic illumination. tures could be analyzed.

\section{Computer Microvision System}

The Computer Microvision system combines a video microscopy system, piezoelectrically focused lenses, a stroboscopic light system, a CCD camera and appropriate electronics including highspeed framegrabbers. Test structures are mounted on the stage of a light microscope (Zeiss Axioplan 2 Motorized Research Microscope, Oberkochen, Germany), resting on a vibration isolation table (Integrated Dynamics Engineering, Woburn MA). Objectives (Zeiss LD-Epiplan 20 and 50) are chosen to achieve high resolution (numerical apertures are 0.4 and 0.6 , respectively) with sufficient working distance $(9.8 \mathrm{~mm}$ and $3.5 \mathrm{~mm}$, respectively) to accommodate external electrical probes. The objectives are attached to the microscope through a piezoelectric focusing unit (PIFOC, Polytec PI, Waldbronn, Germany). Magnified images are projected onto a CCD camera (Pulnix TM-1010), which has $1024 \times 1024$ pixels with $9 \mathrm{~mm}$ spacing. Digitized images from the camera are communicated to a Pentium based computer using a frame grabber (Imaging Technology).

\section{Stroboscopic illumination.}

The maximum frame rate of a digital video imager is typically on the order of tens of Hertz. To image faster motions, we use stroboscopic illumination. A green LED (Nichia NSPG500S, Tokushima 774 , Japan) is pulsed so that the target is illuminated at a selected phase of its motion (see waveforms in Figure 2). Light from the LED is collected with a halogen lamp housing: the halogen lamp was removed, and the LED was mounted near the intended position of the tungsten filament. The plastic lens that originally covered the LED's die was milled away and the flat surface was polished, so that illumination was Köhler.

\section{Modular stimulus generator.}

A single 5 in $\times 10$ in printed circuit card contains all of the circuitry needed to produce the stimulus and timing waveforms (Figure 2). The card contains a high speed 12-bit DAC (Analog Devices AD9713) and a static memory chip ( $64 \mathrm{~K}$ bytes by 16 bits) that is used to hold one cycle of an arbitrary periodic stimulus. The period of the stimulus can be controlled to generate rates from 1 $\mathrm{Hz}$ to $1 \mathrm{MHz}$ with $\mathrm{mHz}$ resolution. The modular stimulus generator also controls the stroboscopic illumination. The amplitude, turn-on time, and turn-off time are under program control. The stimulus

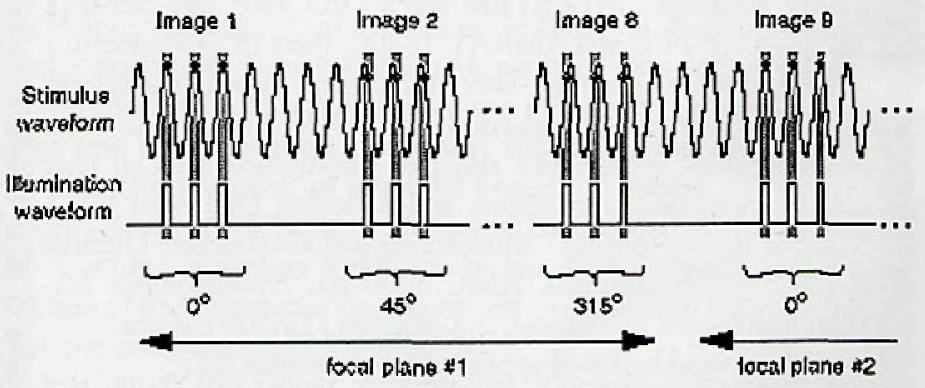

Figure 2: Stroboscopic, volumetric imaging. Motions are driven with a periodic stimulus. The first image is acquired when light from the LED samples the image at times corresponding to peaks in the stimulus waveform. Successive images are acquired at different phases. This process is then repeated for different focal planes selected by a computer-controlled focus adjustment (PIFOC, Polytec PI, Waldbronn, Germany). Magnified images are projected onto a CCD camera (Pulnix TM-1010), which has $1024 \times 1024$ pixels with $9 \mathrm{~mm}$ spacing. Digitized images from the camera are communicated to a Pentium based computer using a frame grabber (Imaging Technology). 
generator can be programmed to sample any number of phases per stimulus period, and arbitrary waits can be programmed to allow transients to decay between stimulus presentations. Stroboscopic illumination can be synchronized to the internally generated stimulus or it can be synchronized to external clocks, which is important for measuring devices that are self-clocked. Since the intensity of the light emitted from an LED is more nearly proportional to current than voltage, the LED is driven by a computercontrolled D/A converter via a voltage-to-current converter. Pulsing is accomplished by switching the current to the LED on and off with a high-speed transistor (ZVN3306A). Because LEDs are fast, the shortest light pulse is limited in practice by the electronics driving the LED (roughly 100 ns for our circuit). Thus, the stroboscopic sampling rate can be as high as $5 \mathrm{MHz}$.

\section{Motion estimates from video images.}

To characterize sinusoidal motions, eight or sixteen images of the specimen are acquired at evenly-spaced phases of the stimulus cycle using stroboscopic illumination. The LED is gated so that the duty cycle is $1 / 8$ period if eight images are collected or $1 / 16$ period if 16 phases are collected. Displacements between images acquired at successive phases are estimated directly from the video images. Although image resolution is limited by the optics to distances on the order of the wavelength of light (typically 525 $\mathrm{nm}$ ), displacements between two such images as small as nanometers can be reliably measured.

\section{D Measurements.}

Silicon devices are opaque to green light. Nevertheless, information about axial motion can be obtained from images obtained from different focal planes. Images from above the best plane of focus of a structure are blurred. As the structure moves axially, the axial blurring pattern moves with the structure. Therefore, the algorithms that are used to track in-plane motions by computing in-plane gradients can equally track out-of-plane motions by computing out-of-plane gradients. Images are acquired at different focal planes to obtain information about 3D motion. In early experiments, focus was adjusted by translating the stage in the axial direction using an integrated stepper motor (part of the Zeiss Axioplan 2 Motorized Research Microscope).This method provided axial positioning with $0.1 \mathrm{~m}$ resolution over large axial excursions-essentially limited only by the rack-and-pinion mechanism that supported the stage. However, axial motions were accompanied by submicrometer in-plane displacements. In later experiments, focus was controlled by translating the objective with a piezoelectric crystal (PIFOC, Polytec PI, Wandbronn, Germany). Although this device limited the maximum axial excursion to 100 $\mathrm{mm}$, in-plane displacements were significantly smaller.

\section{Results}

We have applied Computer Microvision to measure motions of a large variety of surface micromachined and bulk micro-
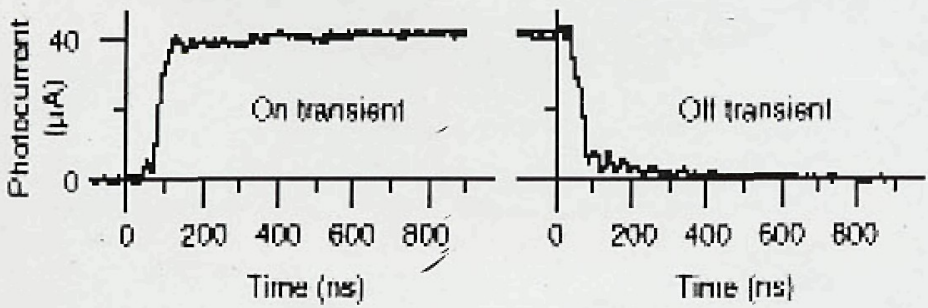

Figure 3: Turn-on and turn-off times of the LED. The signal to the LED was turned on (left) and off (right) and light from the LED was measured using a high-speed photodiode (Motorola MRD500). The traces show the averages of 200 transients. machined MEMS, including lateral resonators, cantilever beams, accelerometers, gyroscopes, diffraction gratings, movable mirrors, variable capacitors, and fatigue test structures. Figure 4 shows representative results for a lateral resonator fabricated by MCNC (Research Triangle Park, NC). Accelerations of the substrate in a direction parallel to the long axes of the teeth in the comb drives (Figure 3 ) cause motions of the shuttle relative to the substrate. These relative motions are resisted by the stiffness of the cantilever system, by the inertia of the shuttle, and by viscous forces in the surrounding air. To quantify these resisting forces, we stimulated motions of the shuttle by applying a sinusoidally varying electrical potential to one comb drive. Motions of the center portion of the shuttle were then determined from sequences of 8 images obtained at evenly spaced phases of the sinusoidal stimulus (Figure 2). Results obtained for 51 frequencies are well approximated by a second-order system with a resonant frequency of $8.86 \mathrm{kHz}$ and a quality of tuning of 13.6 (Figure 5, left panels). The standard deviation of repeated measurements is $5 \mathrm{~nm}$ in magnitude and the signal to noise ratio is nearly $60 \mathrm{~dB}$ at resonance. The standard deviation of repeated phase measurements is less than $1^{\circ}$ except when the magnitude is less than $50 \mathrm{~nm}$.

The Computer Microvision system can also measure motions of the resonator that are orthogonal to the plane of focus. In this device, out-of-plane motions represent failure modes that limit performance. Three dimensional images were obtained by stacking images from 30 focal planes (Figure 5 right panels). Three dimensional motions were obtained by analyzing sequences of 3D images obtained at 8 uniformly spaced phases

\section{Continued on following page}

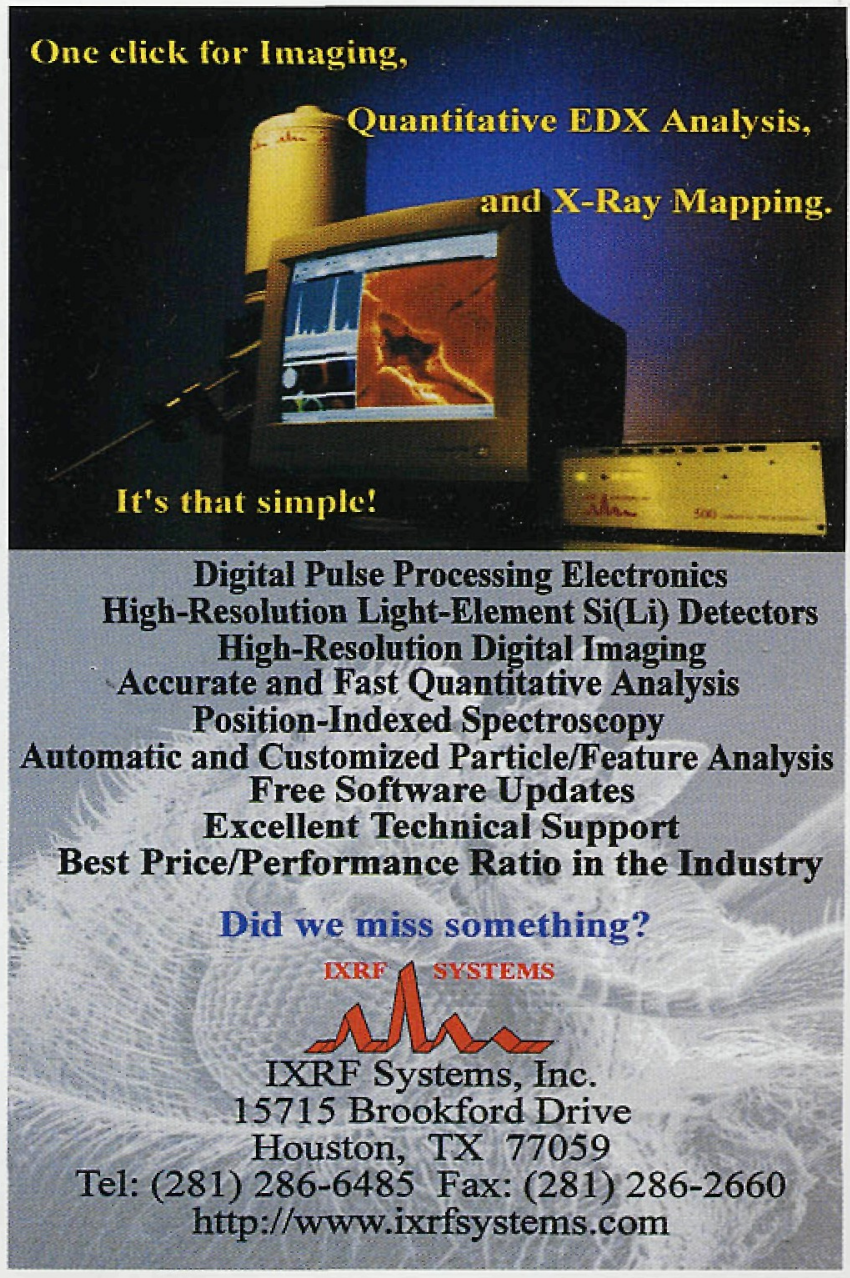




\section{Computer Microvision For MEMS}

Continued from preceding page

of the electrical stimulus. Standard deviations of repeated measurements are $1.1 \mathrm{~nm}$ or less for in-plane measurements and $4.1 \mathrm{~nm}$ for out-of-plane measurements. Apparent motions of the stationary tooth $1.8 \mathrm{~nm}$ or less for in-plane and $5.6 \mathrm{~nm}$ for out-of-plane measurements. Thus, even though the out-of-plane motions are small, they are an order of magnitude larger than the standard deviation of repeated measurements - and are thus well resolved by the measurement system.

\section{Discussion}

We have demonstrated the use of Computer Microvosion as a tool for in situ motion analysis of MEMS. We have illustrated its use to characterize 3D translations of a lateral resonator. These results demonstrate several unique features of Computer Microvision. First, Computer Microvision provides estimates of translations in multiple dimensions. Measurements in multiple dimensions allow unambiguous characterization of complex modes of motion. Second, motion estimates with Computer Microvision are derived directly from sequences of images. Thus, all quantitative results can be checked qualitatively by simply viewing those images. This contrasts with other measurement systems, such as laser interferometry or capacitive sensing, which provide no internal consistency checks.

Results in this paper demonstrate how Computer Microvision can be used to quantify motions of any imaged structure, and to relate motions of multiple structures to characterize complex modes of interaction. Furthermore, the resolution of the system is on the order of nanometers, i.e., less than $1 \%$ of the wavelength of the light used to make the measurement. These features of Computer Microvision hold promise to provide important new opportunities for understanding the mechanical properties of MEMS.

\section{Acknowledgements}

The author acknowledges contributions to this project by C. Quentin Davis, Zoher Z. Karu, Laura Johnson, C. Cameron Abnet, Michael J. Gordon, Stanley S. Hong, Alexander J. Aranyosi, and Michael Mcllrath. This work was supported by grants from DARPA

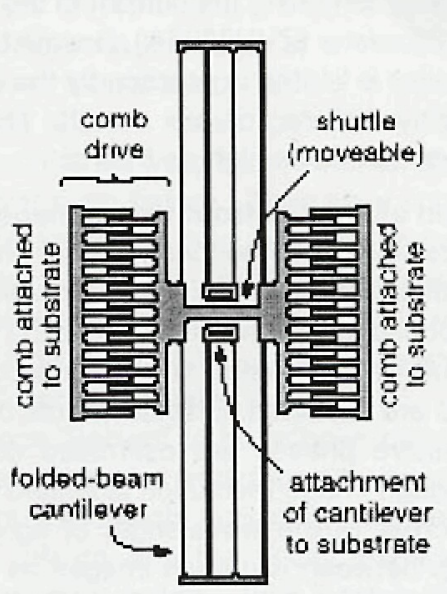

Figure 4: Lateral resonator. A shuttle and associated combs are suspended above the substrate via a folded-beam cantilever spring system. Accelerations of the substrate cause relative motions of the shuttle that can be sensed as a change in capacitance between the interdigitated teeth of combs attached to the shuttle and substrate. The shuttle can also be moved by applying an electrical potential across the interdigitated teeth.
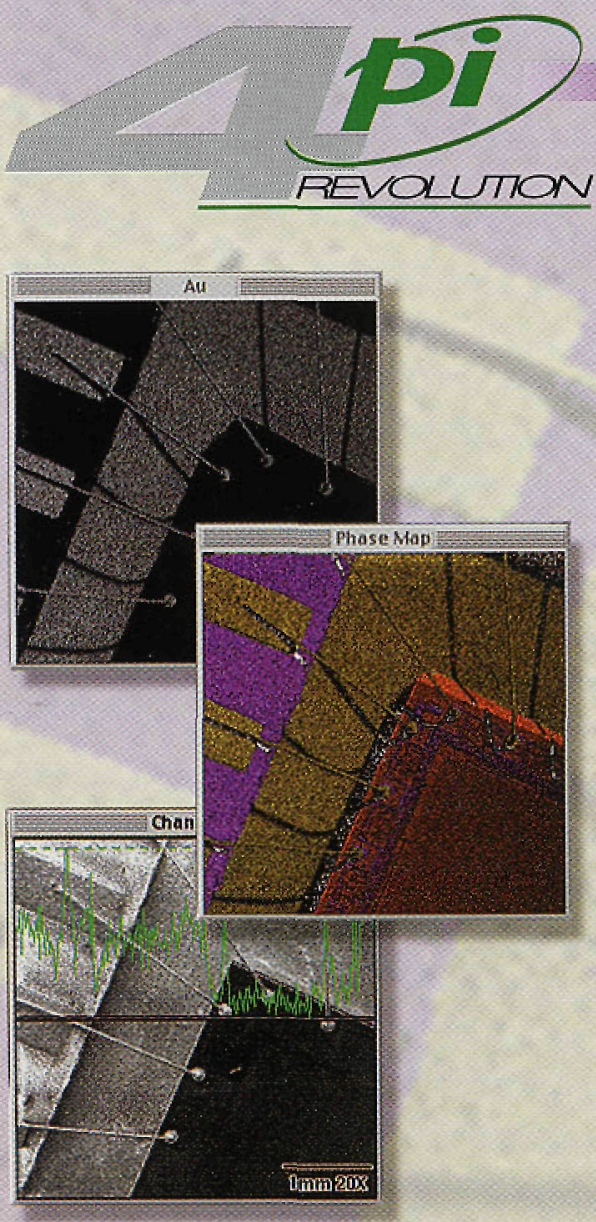

\section{Soectral and Image Acquisition software for Windows ${ }^{\circ}$ and Mac ${ }^{(0)}$ os}

\section{- Micron markers}

-Easy-to-use point-and-click screen tools

- Preview and acquire images of different sizes

- Multiple spectra windows with independent scaling and colors

- Simultaneous acquisition of SEM, WDS, SCA and X-ray images

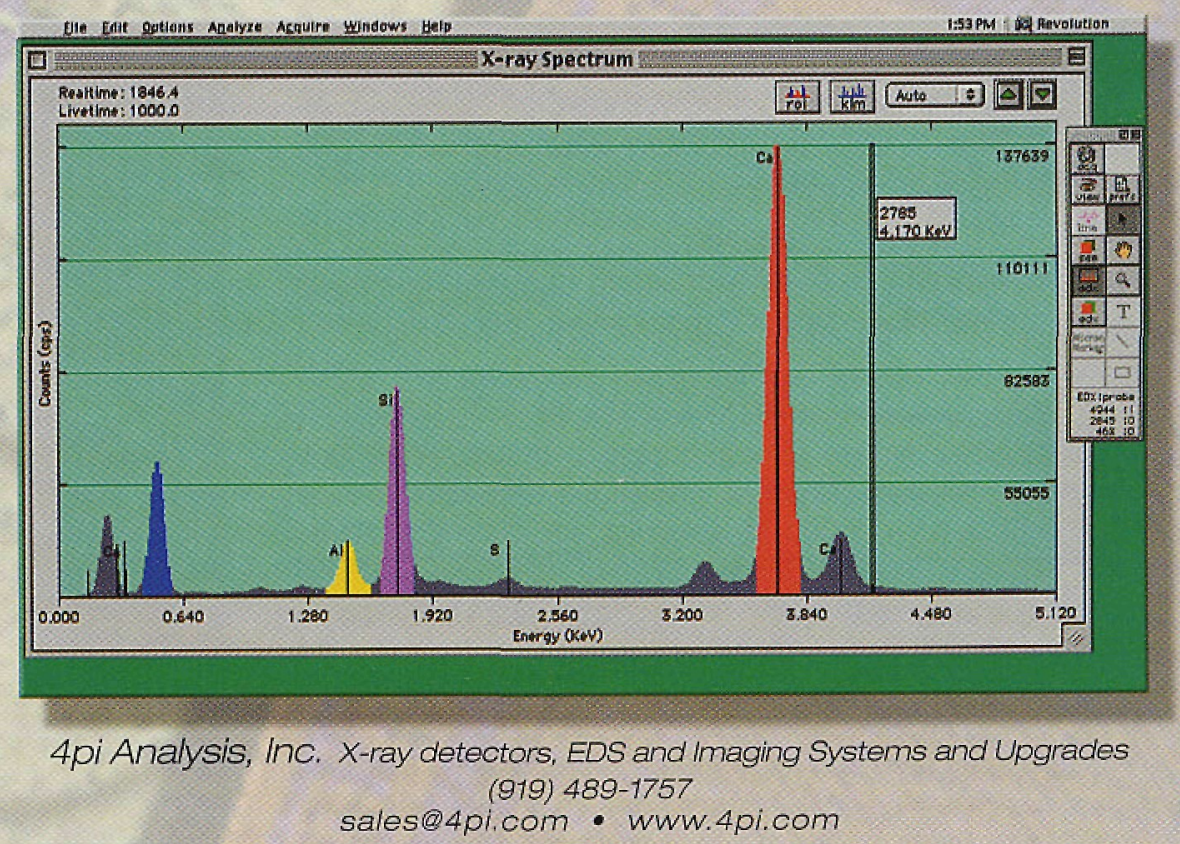


(F30602-97-2-0106) and The Charles Stark Draper Laboratory (E96-414). D. M. Freeman was supported in part by the W. M. Keck Career Development Professorship.

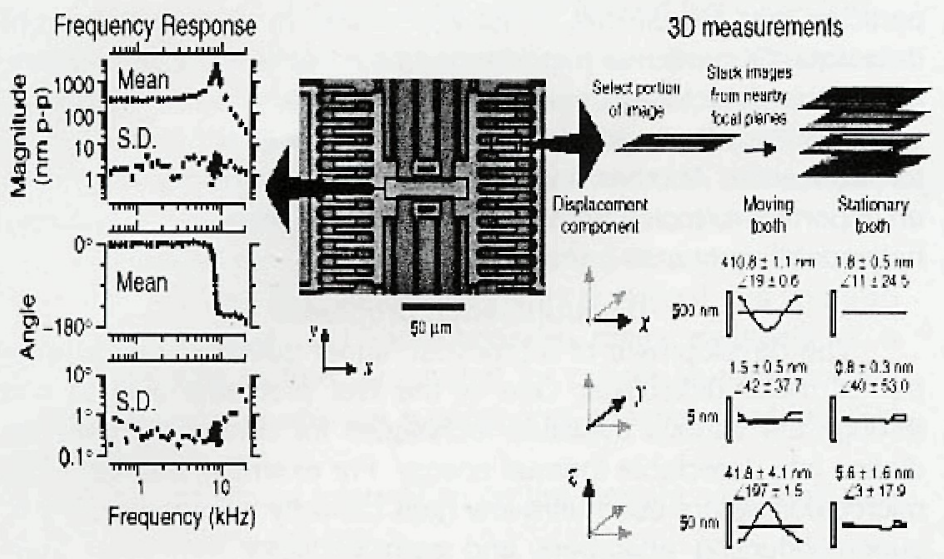

Figure 5: Motions of the lateral resonator. The center panel shows an image of a lateral resonator obtained using our Computer Microvision system. Pixels in a region of interest surrounding the central shuttle were analyzed in images obtained for 8 evenly spaced phases of the sinusoidal stimulus to determine the magnitude and angle of motions in the direction. The left plots show the means and standard deviations (S.D.) of 5 repeated measurements for 51 different frequencies of stimulation. The two comb drives were excited with opposite phases of a sinusoidal (2 $\mathrm{V}$ peak-to-peak) stimulus added to a common $62 \mathrm{~V}$ DC bias. The right panels illustrate $3 \mathrm{D}$ analyses. Regions of interest (white boxes) including a moving and a stationary tooth of the comb drive are shown in pseudo-perspective and displayed with corresponding portions of images taken at focal planes above $(3.8 \mathrm{~m})$ and below $(\mathrm{m})$ the in-focus plane. Similar portions of images from 30 focal planes (separated by $0.545 \mathrm{~m}$ ) and at 8 evenly spaced phases during the stimulus cycle were analyzed to determine displacements in and which are shown in the plots. The numbers indicate the mean standard deviation of the peak-to-peak displacement and angle of the fundamental component of motion for 10 repeated measurements. The two comb drives were excited with opposite phases of a $20 \mathrm{kHz}$ sinusoidal ( $60 \mathrm{~V}$ peak-to-peak) stimulus added to a common $62 \mathrm{VDC}$ bias. The measurement is $5 \mathrm{~nm}$ in magnitude and the signal to noise ratio is nearly $60 \mathrm{~dB}$ at resonance. The standard deviation of repeated phase measurements is less than 1 except when the magnitude is less than 50 $\mathrm{nm}$. The Computer Microvision system can also measure motions of the resonator that are orthogonal to the plane of focus. In this device, out-of-plane motions represent failure modes that limit performance. Three dimensional images were obtained by stacking images from 30 focal planes (Figure 5 right panels). Three dimensional motions were obtained by analyzing sequences of 3D images obtained at 8 uniformly spaced phases of the electrical stimulus. Standard deviations of repeated measurements are $1.1 \mathrm{~nm}$ or less for in-plane measurements and $4.1 \mathrm{~nm}$ for out-ofplane measurements. Apparent motions of the stationary tooth $1.8 \mathrm{~nm}$ or less for in-plane and $5.6 \mathrm{~nm}$ for out-of-plane measurements. Thus, even though the out-of-plane motions are small, they are an order of magnitude larger than the standard deviation of repeated measurements - and are thus well resolved by the measurement system.

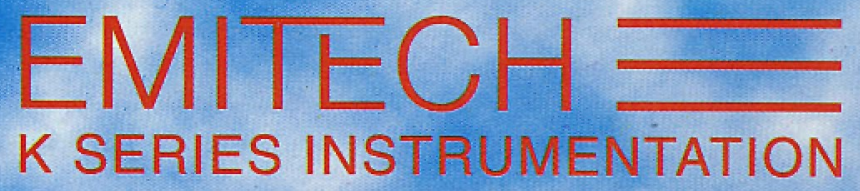

- Extensive range of Consumables \& Accessories - Carbon Evaporation Units

Critical Point Driers

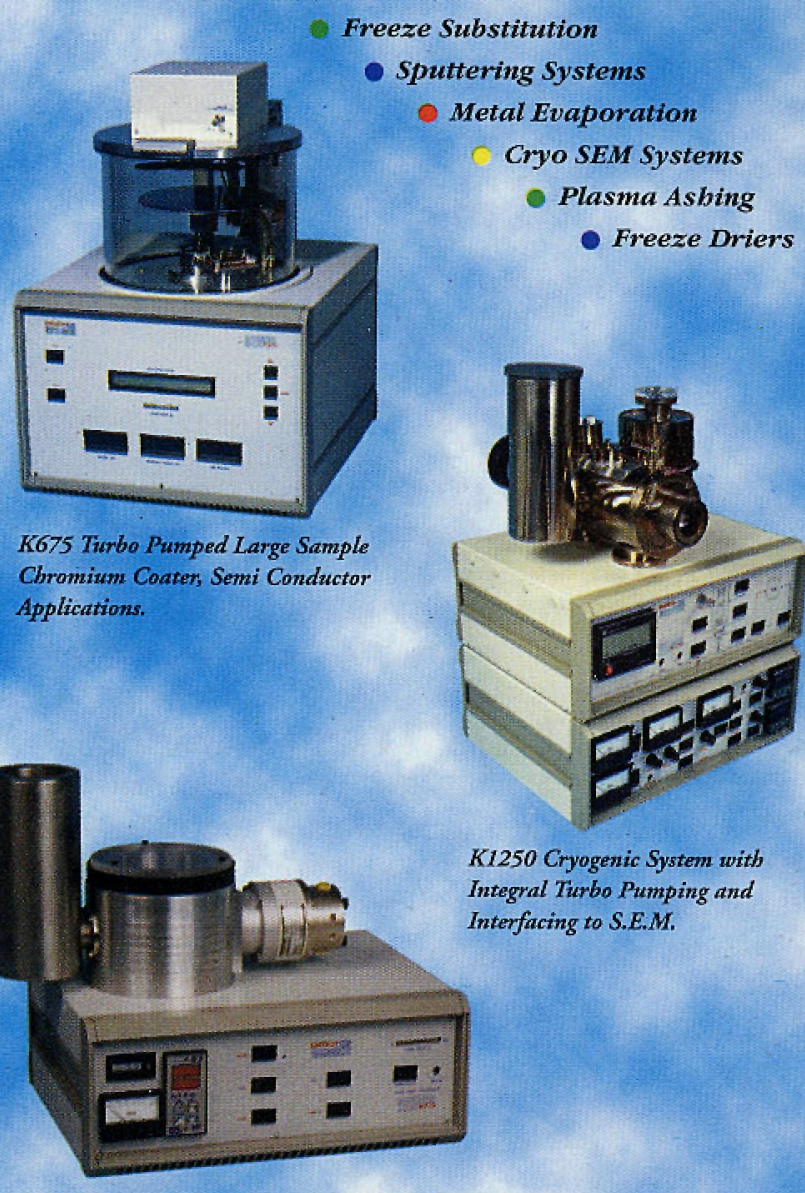

K775 Turbo Pumped Low Temperature Freeze Drien:

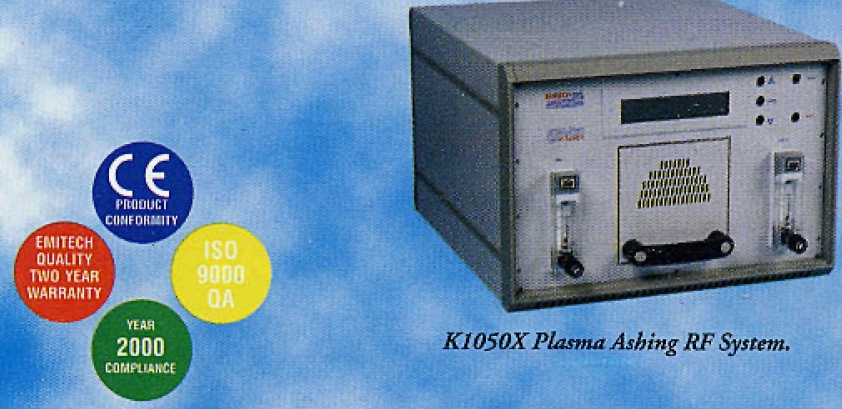

Emitech in U.S.A

Contact: Linda Dailey

888-580-8366

Tel: 2815800568 Fax: 2815800593

\author{
E-mail: emitech@earthlink.net \\ Website: www.emitech.co.uk \\ Emitech Products Inc.
}

